\title{
Generalization of Spin Operators
}

\author{
Moeen Kh. Srour, Munther T. Al-Mokayed, Abdalkarim N. Sahmoud, Mahmoud A. A. Sbaih* \\ Department of Physics, Al-Aqsa University, Gaza, Palestine \\ Email: *ma.sbaih@alaqsa.edu.ps, an.sahmoud@alaqsa.edu.ps, okayed@hotmail.com, moeensrour@hotmail.com
}

How to cite this paper: Srour, M.K., Al-Mokayed, M.T., Sahmoud, A.N. and Sbaih, M.A.A. (2022) Generalization of Spin Operators. Journal of Applied Mathematics and Physics, 10, 132-141.

https://doi.org/10.4236/jamp.2022.101010

Received: December 12, 2021

Accepted: January 18, 2022

Published: January 21, 2022

Copyright $\odot 2022$ by author(s) and Scientific Research Publishing Inc. This work is licensed under the Creative Commons Attribution International License (CC BY 4.0).

http://creativecommons.org/licenses/by/4.0/

\section{Open Access}

\begin{abstract}
The study shall look to the group of generators SU(4). From these generators, a new group spin operator will be constructed. We will classify these groups into right handed groups and left handed groups. These two groups will satisfy all the properties of Pauli spin operators $S_{x}, S_{y}$ and $S_{z}$ with respect to the frame $x y z$. The analysis shows that the number of groups spin operators depends on the order of the group. This leads us to construct the theorem which defines the number of the groups spin operators. The analysis also leads to two kinds of frames: left handed frame (LHF) and right handed frame (RHF). The right handed operators will act on the RHF, and left hand operators act on the LHF. The study shall discuss the notion of spin squeezing for pure spin $3 / 2$ system by using our new frames and new spin operators. It will show that our calculation is equivalent to the calculation by using Pauli spin operators.
\end{abstract}

\section{Keywords \\ Group Generators, Spin Operators}

\section{Introduction}

The wave properties of a particles or the corresponding state can be described only by giving the probability of finding it at particular point with respect to coordinate system.

The collective spin systems are playing very important role in quantum physics [1], because of the symmetric properties of atom or nucleus.

Since the atoms or nucleus deal with a number of identical particles such as electrons or nucleons, it becomes useful to study such collective particle in the shine of group theory.

Quite recently the baryon-baryon interaction in the SU quark model has been studied [2], started from three body problems.

The spin operators possessing has wide range of applications such as squeezing which had been studied [3] [4] [5]. 
The spin squeezing could also be useful for quantum computation [6] [7] [8], quantum simulation [9], and making more precise atom clocks [10] [11] [12] [13].

If $\vec{S}$ denotes the spin angular momentum of a physical system [14], it is known that its components do not commute with each other, but $S^{2}$ commutes with all the components of $\vec{S}\left(S_{x}, S_{y}\right.$ and $\left.S_{z}\right)$. The action of the components of these operators can be on a basis function with respect to the frame $x y z$ that is given by:

$$
\begin{gathered}
\left(S_{x} \pm i S_{y}\right)|s \quad m\rangle=[s(s+1)-m(m \pm 1)]^{\frac{1}{2}}|s, m \pm 1\rangle \\
S^{2}|s \quad m\rangle=s(s+1)|s \quad m\rangle ; \quad s=0, \frac{1}{2}, 1, \cdots \\
S_{z}|s \quad m\rangle=m|s \quad m\rangle ; \quad m=-s,-s+1, \cdots,+s
\end{gathered}
$$

The above equations show that infinite-dimensional Hilbert space of $\vec{S}$ splits into a direct sum of infinite number of Hilbert space.

It is evident in this representation only the matrix $S_{y}$ is purely imaginary, while other matrices are real which is known as Pauli spin matrices with respect to the frame xyz.

Recently in 2020 [15], we had looked to SU(3) generators, from these generators new spin operators for spin 1 system $S_{-x} S_{-y}$ and $S_{-z}$ were constructed. These operators had called left-handed operators with respect to the LHF $x^{\prime} y^{\prime} z$.

In the light of a spin 1 system [15], it becomes relevant to extend the idea to the higher spin system $\left(s=\frac{3}{2}, \cdots\right)$.

The present paper devotes to detailed discussion, the link between the group of generations $\mathrm{SU}(4)$ and spin operators, which are organized as follows:

Section 2 deals with definition of group SU(4). From these generators SU(4), we will construct a new group operators of spin $3 / 2$ system. We shall classify these operators into two classes: right handed operators and left handed operators corresponding to the LHF, and RHF.

In Section 3, we shall look to the squeezing aspect for pure spin $3 / 2$ system by using our new spin group operators.

At the end of discussion, we will show that the new group operators give an equivalent result of squeezing which had been calculated by M. Sbaih [16].

\section{Group Generators SU(4) and Spin Operators}

The Lie algebra and its representations of SU(4) had been studied in detail [17]. As should be clear from the name of $\mathrm{SU}(4)$ is the group of all unitary matrices of order 4 with determinates +1 . It has $4^{2}-1=15$ generators which are usually denoted by $\lambda_{1}, \lambda_{2}, \cdots, \lambda_{15}$.

The group $\mathrm{SU}(4)$ is represented as:

$$
\mathrm{SU}(4)=\left\{A=4 \times 4 \text { complex matrix } \mid A^{+} A=1, \operatorname{det}(A)=1\right\}
$$

The Hermitian matrix generators of SU(4), analogous to Pauli matrices of 
SU(2) which are:

$$
\begin{aligned}
& \lambda_{1}=\left(\begin{array}{llll}
0 & 1 & 0 & 0 \\
1 & 0 & 0 & 0 \\
0 & 0 & 0 & 0 \\
0 & 0 & 0 & 0
\end{array}\right), \lambda_{2}=\left(\begin{array}{cccc}
0 & -i & 0 & 0 \\
i & 0 & 0 & 0 \\
0 & 0 & 0 & 0 \\
0 & 0 & 0 & 0
\end{array}\right), \lambda_{3}=\left(\begin{array}{cccc}
1 & 0 & 0 & 0 \\
0 & -1 & 0 & 0 \\
0 & 0 & 0 & 0 \\
0 & 0 & 0 & 0
\end{array}\right) \\
& \lambda_{4}=\left(\begin{array}{llll}
0 & 0 & 1 & 0 \\
0 & 0 & 0 & 0 \\
1 & 0 & 0 & 0 \\
0 & 0 & 0 & 0
\end{array}\right), \lambda_{5}=\left(\begin{array}{cccc}
0 & 0 & -i & 0 \\
0 & 0 & 0 & 0 \\
i & 0 & 0 & 0 \\
0 & 0 & 0 & 0
\end{array}\right), \lambda_{6}=\left(\begin{array}{cccc}
0 & 0 & 0 & 0 \\
0 & 0 & 1 & 0 \\
0 & 1 & 0 & 0 \\
0 & 0 & 0 & 0
\end{array}\right) \\
& \lambda_{7}=\left(\begin{array}{cccc}
0 & 0 & 0 & 0 \\
0 & 0 & -i & 0 \\
0 & i & 0 & 0 \\
0 & 0 & 0 & 0
\end{array}\right), \lambda_{8}=\frac{1}{\sqrt{3}}\left(\begin{array}{cccc}
1 & 0 & 0 & 0 \\
0 & 1 & 0 & 0 \\
0 & 0 & -2 & 0 \\
0 & 0 & 0 & 0
\end{array}\right), \lambda_{9}=\left(\begin{array}{cccc}
0 & 0 & 0 & 1 \\
0 & 0 & 0 & 0 \\
0 & 0 & 0 & 0 \\
1 & 0 & 0 & 0
\end{array}\right) \\
& \lambda_{10}=\left(\begin{array}{cccc}
0 & 0 & 0 & -i \\
0 & 0 & 0 & 0 \\
0 & 0 & 0 & 0 \\
i & 0 & 0 & 0
\end{array}\right), \lambda_{11}=\left(\begin{array}{cccc}
0 & 0 & 0 & 0 \\
0 & 0 & 0 & 1 \\
0 & 0 & 0 & 0 \\
0 & 1 & 0 & 0
\end{array}\right), \lambda_{12}=\left(\begin{array}{cccc}
0 & 0 & 0 & 0 \\
0 & 0 & 0 & -i \\
0 & 0 & 0 & 0 \\
0 & i & 0 & 0
\end{array}\right) \\
& \left.\lambda_{13}=\left(\begin{array}{cccc}
0 & 0 & 0 & 0 \\
0 & 0 & 0 & 0 \\
0 & 0 & 0 & 1 \\
0 & 0 & 1 & 0
\end{array}\right), \lambda_{14}=\left(\begin{array}{cccc}
0 & 0 & 0 & 0 \\
0 & 0 & 0 & 0 \\
0 & 0 & 0 & -i \\
0 & 0 & i & 0
\end{array}\right), \lambda_{15}=\frac{1}{\sqrt{6}}\left(\begin{array}{cccc}
1 & 0 & 0 & 0 \\
0 & 1 & 0 & 0 \\
0 & 0 & 1 & 0 \\
0 & 0 & 0 & -3
\end{array}\right)\right)
\end{aligned}
$$

These $\lambda_{i}(i=1,2, \cdots, 15)$ matrices are orthogonal and satisfy

$$
\operatorname{Tr}\left(\lambda_{i}\right)^{2}=2, \quad i=1,2, \cdots, 15
$$

From Equation (5) we can construct three spin operators from group generators $\mathrm{SU}(4)$ :

$$
\begin{aligned}
& S_{x}=\lambda_{6} \pm \frac{\sqrt{3}}{2}\left(\lambda_{1} \pm \lambda_{13}\right) \\
& S_{y}=\lambda_{7} \pm \frac{\sqrt{3}}{2}\left(\lambda_{2} \pm \lambda_{14}\right) \\
& S_{z}=\lambda_{3}+\sqrt{3} \lambda_{8}+\sqrt{6} \lambda_{15}
\end{aligned}
$$

It is evident that in this representation only the matrices $S_{y}$ are purely imaginary while $S_{x}$ and $S_{z}$ are real matrices.

From Equations (7) - (9) we construct four groups of spin operators.

The first spin group operators

$$
\left.\begin{array}{l}
S_{1 x}=\lambda_{6}+\frac{\sqrt{3}}{2}\left(\lambda_{1}+\lambda_{13}\right)=\frac{1}{2}\left(\begin{array}{cccc}
0 & \sqrt{3} & 0 & 0 \\
\sqrt{3} & 0 & 2 & 0 \\
0 & 2 & 0 & \sqrt{3} \\
0 & 0 & \sqrt{3} & 0
\end{array}\right) \\
\left.S_{1 y}=\lambda_{7}+\frac{\sqrt{3}}{2}\left(\lambda_{2}+\lambda_{14}\right)=\frac{i}{2}\left(\begin{array}{cccc}
0 & -\sqrt{3} & 0 & 0 \\
\sqrt{3} & 0 & -2 & 0 \\
0 & 2 & 0 & -\sqrt{3} \\
0 & 0 & \sqrt{3} & 0
\end{array}\right), S_{z}=\frac{1}{2}\left(\begin{array}{cccc}
3 & 0 & 0 & 0 \\
0 & 1 & 0 & 0 \\
0 & 0 & -1 & 0 \\
0 & 0 & 0 & -3
\end{array}\right)\right\}
\end{array}\right\}
$$


We have to mention here that $S_{z}$ is the same as for all groups.

The second spin group operators

$$
\left.\begin{array}{c}
S_{2 x}=\lambda_{6}-\frac{\sqrt{3}}{2}\left(\lambda_{1}-\lambda_{13}\right)=\frac{1}{2}\left(\begin{array}{cccc}
0 & -\sqrt{3} & 0 & 0 \\
-\sqrt{3} & 0 & 2 & 0 \\
0 & 2 & 0 & \sqrt{3} \\
0 & 0 & \sqrt{3} & 0
\end{array}\right) \\
S_{2 y}=\lambda_{7}-\frac{\sqrt{3}}{2}\left(\lambda_{2}-\lambda_{14}\right)=\frac{i}{2}\left(\begin{array}{cccc}
0 & \sqrt{3} & 0 & 0 \\
-\sqrt{3} & 0 & -2 & 0 \\
0 & 2 & 0 & -\sqrt{3} \\
0 & 0 & \sqrt{3} & 0
\end{array}\right)
\end{array}\right\}
$$

The third spin group operators

$$
\left.\begin{array}{c}
S_{3 x}=\lambda_{6}+\frac{\sqrt{3}}{2}\left(\lambda_{1}-\lambda_{13}\right)=\frac{1}{2}\left(\begin{array}{cccc}
0 & \sqrt{3} & 0 & 0 \\
\sqrt{3} & 0 & 2 & 0 \\
0 & 2 & 0 & -\sqrt{3} \\
0 & 0 & -\sqrt{3} & 0
\end{array}\right) \\
S_{3 y}=\lambda_{7}+\frac{\sqrt{3}}{2}\left(\lambda_{2}-\lambda_{14}\right)=\frac{i}{2}\left(\begin{array}{cccc}
0 & -\sqrt{3} & 0 & 0 \\
\sqrt{3} & 0 & -2 & 0 \\
0 & 2 & 0 & \sqrt{3} \\
0 & 0 & -\sqrt{3} & 0
\end{array}\right)
\end{array}\right\}
$$

The fourth spin group operators

$$
\left.\begin{array}{c}
S_{4 x}=\lambda_{6}-\frac{\sqrt{3}}{2}\left(\lambda_{1}+\lambda_{13}\right)=\frac{1}{2}\left(\begin{array}{cccc}
0 & -\sqrt{3} & 0 & 0 \\
-\sqrt{3} & 0 & 2 & 0 \\
0 & 2 & 0 & -\sqrt{3} \\
0 & 0 & -\sqrt{3} & 0
\end{array}\right) \\
S_{4 y}=\lambda_{7}-\frac{\sqrt{3}}{2}\left(\lambda_{2}+\lambda_{14}\right)=\frac{i}{2}\left(\begin{array}{cccc}
0 & \sqrt{3} & 0 & 0 \\
-\sqrt{3} & 0 & -2 & 0 \\
0 & 2 & 0 & \sqrt{3} \\
0 & 0 & \sqrt{3} & 0
\end{array}\right)
\end{array}\right\}
$$

The commutation relations for these groups operators can be worked out and are found to be:

$$
\begin{gathered}
{\left[S_{1 x}, S_{1 y}\right]=\left[S_{2 x}, S_{2 y}\right]=\left[S_{3 x}, S_{3 y}\right]=\left[S_{4 x}, S_{4 y}\right]=i S_{z}} \\
{\left[S_{1}^{2}, S_{1+}\right]=\left[S_{2}^{2}, S_{2+}\right]=\left[S_{3}^{2}, S_{3+}\right]=\left[S_{4}^{2}, S_{4+}\right]=0} \\
{\left[S_{1}^{2}, S_{1-}\right]=\left[S_{2}^{2}, S_{2-}\right]=\left[S_{3}^{2}, S_{3-}\right]=\left[S_{4}^{2}, S_{4-}\right]=0}
\end{gathered}
$$

and

$$
\left.\begin{array}{l}
{\left[S_{z}, S_{1 \pm}\right]= \pm S_{1 \pm},\left[S_{z}, S_{2 \pm}\right]= \pm S_{2 \pm}} \\
{\left[S_{z}, S_{3 \pm}\right]= \pm S_{3 \pm},\left[S_{z}, S_{4 \pm}\right]= \pm S_{4 \pm}}
\end{array}\right\}
$$


In the light of the above studies, it is clear that in the case of SU(4) group, we have constructed 4-groups spin operators $(s=3 / 2)$, one of them is a Pauli operators. Although it had been shown that in the case of $\mathrm{SU}(2)(s=1 / 2)$ possesses one group generators. For SU(3) [15] there are two groups spin operators. It's clear that the number of groups spin operators is increasing as the order of group increases.

\section{Theorem: To Every Group Generator SU(n) There Are $2^{n-2}$ Spin Groups Operators}

It may also note here that the first group $S_{1}$ and the third group $S_{3}$ are giving the same result in a physical application, as we shall show in the next section. The groups $S_{2}$ and $S_{4}$ also will give the same result in physical calculation.

It is clear also that the four spin groups operators $S_{1}, S_{2}, S_{3}$, and $S_{4}$ are coincide with the same $z$-axis as shown in Figure 1.

Quite recently [15], we had shown that in the case of a spin 1 system $(s=1)$, it will possess two groups spin operators. We had classified these two group to a left handed groups and right handed groups.

Our analysis shows that a spin $3 / 2$ system possesses four spin groups operators $S_{1}, S_{2}, S_{3}$, and $S_{4}$.

Let us consider spin $3 / 2$ state $|\psi\rangle$ making an angle $\theta$ with respect to $\mathrm{z}$-axis in the frame $x_{1} y_{1} z_{1}$ and the corresponding operators $S_{1 x} S_{1 y}$ and $S_{1 z}$ as shown in Figure 1.

In the frame $x_{2} y_{2} z_{2}$ where $z_{2}$ coincide with $z_{1}$ in a frame $x_{1} y_{1} z_{1}$ the angel will be $\theta+\pi$ and the phase angle $\varphi$. The third frame $x_{3} y_{3} z_{3}$ the $z_{3}$ will be in the same direction of $Z_{1}$ and the spin operators $S_{1}$ and $S_{3}$ having the same physical calculation, therefore $S_{1}$ and $S_{3}$ will act on the same frame we shall call this frame $x y z$.

The frames $x_{2} y_{2} Z_{2}$ and $x_{4} y_{4} z_{4}$ are having the same $z$-direction of $z_{1}$ and the same physical result, therefore we shall call the second frame $x^{\prime} y^{\prime} z$ '.

The four spin groups operator will act in two frames. These two frames are called RHF and LHF.

The spin group operators acting on the RHF called right handed spin operator, yield the operator acting on the LHF called left handed spin operator.

An advantage of RHF, LHF and spin operators in the nuclear physics. The interaction happens between the particle and antiparticle such as:

$$
\mathrm{e}^{+}+\mathrm{e}^{-} \rightarrow 2 h v \quad(\gamma \text {-radiation })
$$

In such process the electron and positron are coincide with one axis of motion.

\section{Squeezing Criterion for Pure Spin 3/2 System}

The notion of squeezing had been discussed for spin system with arbitrary but sharp spin value by several authors [18]-[24].

Recently [15], the squeezing criterion for a spin 1 system had been discussed by using the left-handed operators. 

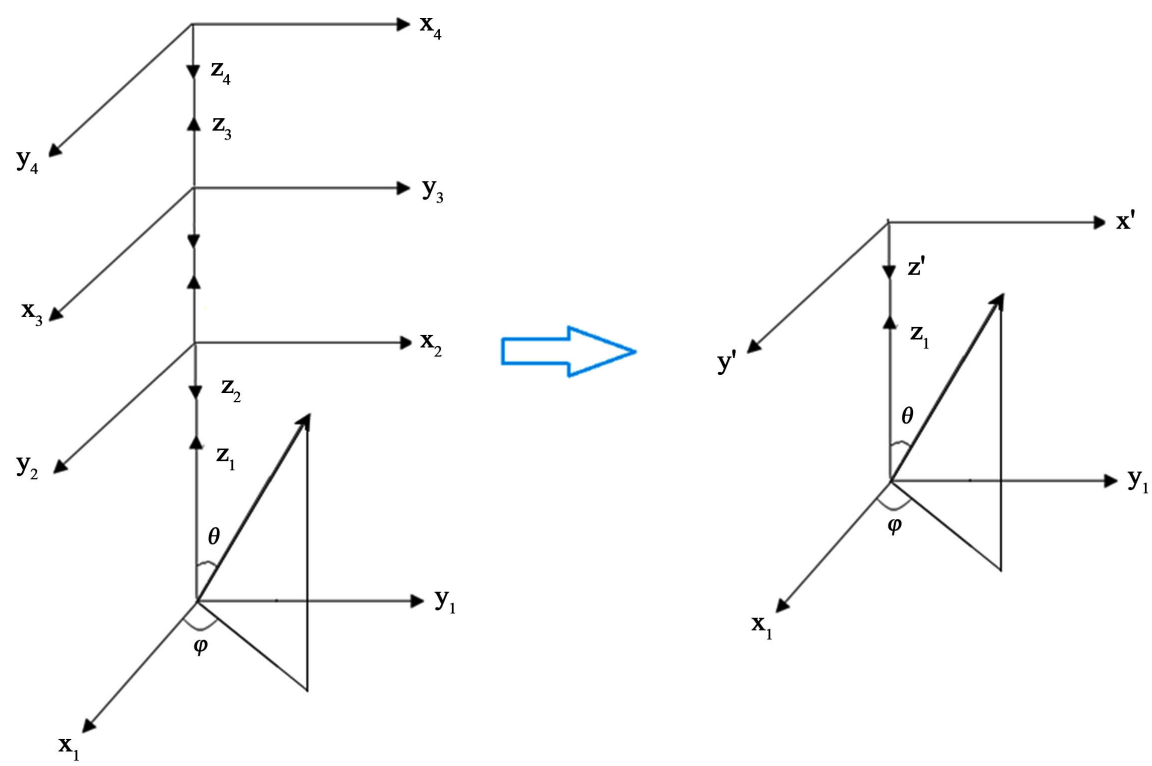

Figure 1 . The spin vector $(s=3 / 2)$ with respect to the frames $x_{i} y_{i} Z_{i}(i=1,2,3,4)$.

In this section we deal with the squeezing aspects for pure spin $3 / 2$ system by using the construction spin operators and frames.

The squeezing condition restrict to Heisenberg's relationship, that a spin $S$ state is squeezed in the spin component normal to the mean spin direction with respect to the (RHF) and spin operators if:

$$
\Delta S_{i x}^{2}<\frac{\left|\left\langle S_{z}\right\rangle\right|}{2}, \quad i=1,3, \cdots
$$

or

$$
\Delta S_{i y}^{2}<\frac{\left|\left\langle S_{z}\right\rangle\right|}{2}, \quad i=1,3, \cdots
$$

A normalized pure state of $s=3 / 2$ system, can be expressed in terms of angular momentum state: $\left|\frac{3}{2} \frac{3}{2}\right\rangle,\left|\frac{3}{2} \frac{1}{2}\right\rangle,\left|\frac{3}{2}-\frac{1}{2}\right\rangle,\left|\frac{3}{2}-\frac{3}{2}\right\rangle$ with respect to the RHF $x y z$ and right handed operators $S_{+1}$ and $S_{+3}$ with a non-zero mean spin direction $\left\langle S_{z}\right\rangle$ can be written in the matrix form [16]:

$$
|\psi\rangle=\left(\begin{array}{c}
\sin \theta \\
0 \\
\cos \theta \mathrm{e}^{+i \varphi} \\
0
\end{array}\right)
$$

The above equation is satisfying:

$$
\left\langle S_{1 x}\right\rangle=\left\langle S_{3 x}\right\rangle=\left\langle S_{1 y}\right\rangle=\left\langle S_{3 y}\right\rangle=0
$$

Which happen to be a Lakin frame with respect to RHF or LHF.

In the RHF $x y z$, the relevant quantities need for studying the squeezing turn out to be:

$$
\Delta S_{1 x}^{2}=\Delta S_{3 x}^{2}=\frac{1}{4}\left[3+4 \cos ^{2} \theta+4 \sqrt{3} \sin \theta \cos \theta \cos \varphi\right]
$$


or

$$
\Delta S_{1 y}^{2}=\Delta S_{3 y}^{2}=\frac{1}{4}\left[3+4 \cos ^{2} \theta-4 \sqrt{3} \sin \theta \cos \theta \cos \varphi\right]
$$

and

$$
\left\langle S_{1 z}\right\rangle=\left\langle S_{3 z}\right\rangle=\frac{1}{4}\left[3-4 \cos ^{2} \theta\right]
$$

So that the squeezing conditions corresponding to the right handed operators $S_{1}$ and $S_{3}$ with respect to the RHF $x y z$

$$
3+4 \cos ^{2} \theta+4 \sqrt{3} \sin \theta \cos \theta \cos \varphi<\left|3-4 \cos ^{2} \theta\right|
$$

or

$$
3+4 \cos ^{2} \theta-4 \sqrt{3} \sin \theta \cos \theta \cos \varphi<\left|3-4 \cos ^{2} \theta\right|
$$

It's clear from the above Equations (24-28) that the two groups right handed operators gives the same result, this implies that the Pauli spin operators are not unique in the same frame.

Let us now look to the squeezing with respect to a LHF $x^{\prime} y^{\prime} z^{\prime}$ and left handed operators $S_{2}$ and $S_{4}$. We notice from Figure 1 that the polar angles $\theta$ with respect to the LHF are $\theta+\pi$.

The state $|\psi\rangle$ with respect to the LHF $x^{\prime} y^{\prime} z^{\prime}$ in the matrix form

$$
|\psi\rangle=\left(\begin{array}{c}
\sin (\theta+\pi) \\
0 \\
\cos (\theta+\pi) \mathrm{e}^{i \varphi} \\
0
\end{array}\right)
$$

It is clear from the above Equation (29)

$$
\left\langle S_{2 x^{\prime}}\right\rangle=\left\langle S_{4 x^{\prime}}\right\rangle=\left\langle S_{2 y^{\prime}}\right\rangle=\left\langle S_{4 y^{\prime}}\right\rangle=0
$$

which happens to be a Lakin frame.

For such state, the relevant quantities indeed necessary for studying the squeezing with respect to LHF and left handed operators are turn out to be:

$$
\Delta S_{2 x^{\prime}}^{2}=\Delta S_{4 x^{\prime}}^{2}=\frac{1}{4}\left[3+4 \cos ^{2} \theta-4 \sqrt{3} \sin \theta \cos \theta \cos \varphi\right]
$$

or

$$
\begin{gathered}
\Delta S_{2 y^{\prime}}^{2}=\Delta S_{4 y^{\prime}}^{2}=\frac{1}{4}\left[3+4 \cos ^{2} \theta+4 \sqrt{3} \sin \theta \cos \theta \cos \varphi\right] \\
\left\langle S_{2 z}\right\rangle=\left\langle S_{1 z}\right\rangle
\end{gathered}
$$

The squeezing conditions with respect to the LHF and left handed operators

$$
3+4 \cos ^{2} \theta-4 \sqrt{3} \sin \theta \cos \theta \cos \varphi<\left|3-4 \cos ^{2} \theta\right|
$$

or

$$
3+4 \cos ^{2} \theta+4 \sqrt{3} \sin \theta \cos \theta \cos \varphi<\left|3-4 \cos ^{2} \theta\right|
$$


It is clear from the above calculations that by using the left handed operators ( $S_{2}$ and $S_{4}$ ) or right handed operators $\left(S_{1}\right.$ and $S_{3}$ ), we get the same result [16], by using Pauli spin operators.

\section{Conclusions}

The study establishes the link between the group generators and quantum spin operators.

The matrices of Lie algebra of $\mathrm{SU}(4)$ has been represented. From the group generators SU(4), we had constructed new groups of spin operator. These two groups are classified into two classes left handed groups and right handed groups. Our analysis shows that the number of group operators depends on the order of a group $n$. It has been shown by Moeen [15] that, a spin $1 / 2$ system having 1 group spin operators, while in a spin 1 system has two groups of spin operators, but in the spin $3 / 2$ system, it is having four groups spin operators. This implies that the number of groups operators increases with the increase of the ordered of group.

A new theorem which defines the number of groups spin operators has been formed.

These new groups spin operators lead us also to two kinds of frames, LHF and RHF.

The squeezing of a pure spin $3 / 2$ system has been studied by using LHF, RHF and a new spin operator. Our calculation shows equivalent result and same values which are done by M A. A. Sbaih [16].

This work is promising and could expand to cover a higher order of groups spin operators. The RH and LH frames and operators could be useful in studying the interaction between particles and antiparticles.

\section{Conflicts of Interest}

The authors declare no conflicts of interest regarding the publication of this paper.

\section{References}

[1] Perlin, M.A. and Rey, A.M. (2020) Short-Time Expansion of Heisenberg Operators in Open Collective Quantum Spin Systems. Physical Review A, 101, Article ID: 023601.

[2] Fujiwara, Y., Suzuki, Y. and Nakamoto, C. (2006) Baryon-Baryon Interactions in the SU6 Quark Model and Their Applications to Light Nuclear Systems. Progress in Particle and Nuclear Physics, 58, 439-520.

[3] Guehne, O. and T'oth, G. (2009) Entanglement Detection. Physics Reports, 474, 1-75. https://doi.org/10.1016/j.physrep.2009.02.004

[4] Amico, L., Fazio, R., OSterloh, A. and Vedral, V. (2008) Entanglement in Many-Body Systems. Reviews of Modern Physics, 80, 517-576. https://doi.org/10.1103/RevModPhys.80.517

[5] Horodecki, R., Horodecki, P., Horodecki, M. and Horodecki, K. (2009) Quantum 
Entanglement. Reviews of Modern Physics, 81, 865-942. https://doi.org/10.1103/RevModPhys.81.865

[6] Buluta, I., Ashhab, S. and Nori, F. (2011) Natural and Artificial Atoms for Quantum Computation.

[7] You, J.Q. and Nori, F. (2005) Superconducting Circuits and Quantum Information. Physics Today, 58, 42-47. https://doi.org/10.1063/1.2155757

[8] Shevchenko, S.N., Ashhab, S. and Nori, F. (2010) Landau-Zener Stuckelberg interferometry. Physics Reports, 492, 1-30. https://doi.org/10.1016/j.physrep.2010.03.002

[9] Buluta, I. and Nori, F. (2009) Quantum Simulators. Science, 326, 108-111. https://doi.org/10.1126/science.1177838

[10] Leroux, I.D., Schleier-Smith, M.H. and Vuletic, V. (2010) Orientation Dependent Entanglement Lifetime in Squeezed Atomic Clock. Physical Review Letters, 104, Article ID: 250801. https://doi.org/10.1103/PhysRevLett.104.250801

[11] Andre, A., Sorensen, A. and Lukin, M. (2004) Stability of Atomic Clocks Based on Entangled Atoms. Physical Review Letters, 92, Article ID: 230801. https://doi.org/10.1103/PhysRevLett.92.230801

[12] Meiser, D., Ye, J. and Holland, M.J. (2008) Spin Squeezing in Optical Lattice Clocks via Lattice-Based QND Measurements. New Journal of Physics, 10, Article ID: 073014. https://doi.org/10.1088/1367-2630/10/7/073014

[13] Oblak, D., Petrov, P., Garrido Alzar, C., Tittel, W., Vershovski, A., Mikkelsen, J., Sorensen, J. and Polzik, E. (2005) Quantum Noise-Limited Interferometric Measurement of Atomic Noise: Towards Spin Squeezing on Yhe Cs Clock Transition. Physical Review A, 71, Article ID: 043807. https://doi.org/10.1103/PhysRevA.71.043807

[14] Schiff, L.I. (1955) Quantum Mechanics. McGraw-Hill, New York.

[15] Srour, M.Kh., Sahmoud, A.N., Al-Mokayed, M.T., Sbaih, M.A.A. and Sumour, M.A. (2020) Generation of Spin 1 System. Journal of Applied Mathematics and Physics, 8 , 1706-1714. https://doi.org/10.4236/jamp.2020.89128

[16] Sbaih, M.A.A., Srour, M.Kh. and Sahmoud, A.N. (2020) Squeezing and Correlation of Pure Spin 3/2 System. Al-Aqsa University Journal (Natural Sciences Series), 22, 114-124.

[17] Sbaih, M.A.A., Srour, M.K.H., Hamada, M.S. and Fayad, H.M. (2013) Lie Algebra and Representation of SU(4). Electronic Journal of Theoretical Physics: EJTP, 10, 9-26.

[18] Kitagawa, M. and Ueda, M. (1993) Squeezed Spin States. Physical Review A, 47, 5138-5143. https://doi.org/10.1103/PhysRevA.47.5138

[19] Puri, R.R. (1997) Coherent and Squeezed States on Physical Basis. Pramana, 48, 787-797. https://doi.org/10.1007/BF02845612

[20] Wineland, D.J., Bollinger, J.J., Itano, W.M., Moore, F.L. and Heinzeen, D.J. (1992) Spin Squeezing and Reduced Quantum Noise in Spectroscopy. Physical Review A, 46, R6797. https://doi.org/10.1103/PhysRevA.46.R6797

[21] Agarwal, G.S. and Puri, R.R. (1993) Atomic States with Spectroscopic Squeezing. Physical Review A, 49, 4968-4971. https://doi.org/10.1103/PhysRevA.49.4968

[22] Mallesh, K.S., Sirsi, S., Sbaih, M.A.A., Deepak, P.N. and Ramachandran, G. (2000) Squeezing and Non-Oriented Spin States. Journal of Physics A: Mathematical and General, 33, 779. https://doi.org/10.1088/0305-4470/33/4/310

[23] Mallesh, K.S., Sirsi, S., Sbaih, M.A.A., Deepak, P.N. and Ramachandran, G. (2001) 
Spin Squeezing of Mixed Systems. Journal of Physics A: Mathematical and General, 34, 3293.

[24] Mallesh, K.S., Sirsi, S., Sbaih, M.A.A., Deepak, P.N. and Ramachandran, G. (2000) Spin Squeezing in Nucleon-Nucleon Scattering. International Symposium on Nuclear Physics, Bhabha Atomic Research Centre, 43B, 360. 